\title{
Stability of a Hot Smoluchowski Fluid
}

\author{
R. F. Streater, \\ Dept. of Mathematics, \\ King's College, \\ Strand, London WC2R 2LS. \\ ray.streater@kcl.ac.uk \\ http:// www.mth.kcl.ac.uk/ ${ }_{\text {streater/ }}$
}

$26 / 10 / 2000$

\begin{abstract}
We study coupled nonlinear parabolic equations for a fluid described by a material density $\rho$ and a temperature $\Theta$, both functions of space and time. In one dimension, we find some stationary solutions corresponding to fixing the temperature on the boundary, with no-escape boundary conditions for the material. For the special case, where the temperature on the boundary is the same at both ends, the linearized equations for small perturbations about a stationary solution at uniform temperature and density are derived; they are subject to boundary conditions, Dirichlet for $\Theta$ and no-flow conditions for the material.

The spectrum of the generator $L$ of time evolution, regarded as an operator on $L^{2}[0,1]$, is shown to be real, discrete and non-positive, even though $L$ is not self-adjoint. This result is necessary for the stability of the stationary state, but might not be sufficient. The problem lies in the fact that $L$ is not a sectorial operator, since its numerical range is $\mathbf{C}$.
\end{abstract}

\section{Introduction}

In [15, 16, 17, 18, 19] we derived systems of parabolic partial differential equations as the diffusion limit of discrete stochastic models describing a fluid of identical particles moving in an external potential $V(x)$. In the continuum limit the material is described by a density $\rho(x, t)$ and the internal energy is described by a temperature field $\Theta(x, t)$. The various models are 
based on different kinematic assumptions, and this shows up in the details of the equations of evolution. In [15, 19] there is a maximum possible value of the density, called $\rho_{\max }$, corresponding to the state in which all the sites of the discrete model are occupied. We showed that the first and second laws of thermodynamics hold in these models, and that in each case, the system has the Onsager form [15, 20], though it is not linear and not near equilibrium. The difference between [19] and [15] is that in the latter, the hopping probability in the underlying discrete model grows proportionally with the energy of the state the particle occupies. This assumption leads to a surprising phenomenon in the continuum limit: the model shows the Soret and Dufour effects. This had been derived in 1912 by Enskog [11] and in 1912 by Chapman [10] from the Boltzmann equation, but only for gas mixtures.

These and similar models have been extended and studied by Biler, Dolbeault, Esteban, Hebisch, Karch, Krzywicki, Markowich and Nadzieja in a series of papers [2, 3, $4,5,6,0,0,8,9,13,14]$. In these papers, $V$ is either an external potential or is the mutual gravitational or electrical potential between the particles, and so obeys a Helmholtz equation with $\rho(x, t)$ as its source. This leads to an integro-differential system, for which the authors have been able to prove existence and uniqueness of solutions in many cases, and to study the asymptotic return to equilibrium.

In this paper we concentrate on the model [15] with the Soret and Dufour effects. The heat capacity per particle is unity, and so the energy density is naturally defined to be

$$
\mathcal{E}(x, t):=\rho(x, t)[\Theta(x, t)+V(x)] .
$$

The dynamics conserves the total number of particles and the total energy, and this leads to the local conservation laws:

$$
\begin{aligned}
& \frac{\partial \rho}{\partial t}+\operatorname{div} j_{c}=0 \\
& \frac{\partial \mathcal{E}}{\partial t}+\operatorname{div} j_{e}=0 .
\end{aligned}
$$

The material current $j_{c}$ and the energy current $j_{e}$ of the model are shown to be

$$
\begin{aligned}
& j_{c}=-\lambda\left(\Theta \nabla \rho+\rho\left(1-\rho / \rho_{\max }\right) \nabla(\Theta+V)\right), \\
& j_{e}=2\left(\Theta j_{c}-\lambda \rho\left(1-\rho / \rho_{\max }\right) \Theta \nabla \Theta\right)+V j_{c} .
\end{aligned}
$$


For rare gases, the density is much less than its maximum, and the system of equations simplifies. The currents for material and energy are then

$$
\begin{aligned}
& j_{c}=-\lambda(\Theta \nabla \rho+\rho \nabla(\Theta+V)) \\
& j_{e}=2\left(\Theta j_{c}-\lambda \rho \Theta \nabla \Theta\right)+V j_{c} .
\end{aligned}
$$

The derivation given in [15] starts with a Markov chain, and takes the continuum limit. The equations are therefore local in time, and if $V$ is timedependent, the same calculation shows that the right-hand side of eq. (3) should be $\rho \partial V / \partial t$. The model falls into the general scheme advocated by Wojnar 21].

In [15] we show that the entropy is an increasing function of time (except at a stationary configuration); thus the model obeys both the first and the second law of thermodynamics if the boundary conditions allow no material or heat to enter or leave the system. The entropy is shown to be

$$
\begin{aligned}
S & =-\int \rho(x) \log \rho(x) d x-\int\left(\rho_{\max }-\rho(x)\right) \log \left(1-\rho / \rho_{\max }\right) d x+ \\
& +\int \rho(x) \log \Theta(x) d x .
\end{aligned}
$$

For small $\rho(x) / \rho_{\max }$, so that we can ignore $\left(\rho / \rho_{\max }\right)^{2}$, this simplifies to

$$
S=-\int \rho(x) \log \rho(x) d x+\int \rho(x) \log \Theta(x) d x
$$

apart from the term $\int \rho(x) d x$, which is constant in time if no material enters or leaves the system.

Usually, the energy-balance equation (3) is ignored, and the temperature put equal to $\Theta_{0}$ for all space and time (the iso-thermal situation), and we reduce to the Smoluchowski equation for the Brownian particle in a potential. We avoid this simplification, which loses the Soret and Dufour effects that come from the cross-terms $\nabla \Theta$ in eq. (16) and $2 \Theta j_{c}$ in $j_{e}$. The first is the Joule-Thompson effect (in the local form called the Soret effect), which ensures that a gradient in the temperature causes a flow of material, even when the density is constant. The term $2 \Theta j_{c}$ is the Dufour effect, which means that there is a flow of heat even when the temperature is constant, if there is a non-zero particle current. We might expect the flow of heat due to convection to be $\Theta j_{c}$ since the heat capacity is unity, and the factor 2 was a surprise. In [15] it was shown that the other half of the contribution 
$2 \Theta j_{c}$ is needed as the Onsager dual of the Soret term, $\nabla \Theta$, which occurs in $j_{c}$.

In this paper, we study these equations in one dimension in a bounded interval, which without loss of generality may be taken to be $[0,1]$. We shall continue to write $\nabla$ for the gradient, so for the rest of the paper, $\nabla=\partial / \partial x$.

Consider the the boundary conditions $\Theta=\Theta_{0}$ for all time at $x=0$ and $x=1$, and $j_{c}=0$ for all time at $x=0$ and $x=1$. These have the direct physical meaning that the system is in a heat-bath at temperature $\Theta_{0}$, and no material escapes through the boundary, which is impermeable to the particles of the fluid, but conducts heat perfectly. Such mixed DirichletNeumann conditions for systems do not appear to have been considered before in that it leads to a new type of generator $L$ of the semigroup of time-evolution, for which form techniques do not work. This is because the operator $L$ is not sectorial.

It is obvious that there is a stationary solution satisfying the following conditions: $\Theta(x, t)=\Theta_{0}$, and $j_{c}(x, t)=0$ for all space and time. It is then easy to solve for $\rho$ in the case of a constant gravitational field (§2). We also briefly discuss the case when $\Theta$ is not constant.

In $\S 3$ we study the stability of small perturbations about the stationary state, by the usual method. We limit the consideration to the case $V=0$, and so leave for the future the (Bénard) instabilities expected for a liquid in a gravitational field heated from below and cooled from above.

We give a proof that the eigenvalues are real and non-positive, by exhibiting a $2 \times 2$ similarity transform (not unitary) that transforms the operator $L$ to a diagonal positive matrix, times the known negative self-adjoint operator, the Laplacian with self-adjoint boundary conditions. This shows that $L$ is closed, and leads to the general study of operators of this type, which has been pursued by L. Boulton. The new difficulty is that the numerical range of the generator is the whole complex plane.

\section{Some No-flow Solutions}

Let $j_{c}$ and $j_{e}$ be given by eq. (6) and (7). Let us impose the no-flow conditions $\operatorname{div} j_{c}=0$, so in one dimension, $j_{c}$ is a constant. This must be zero, since we impose the boundary condition of no material flow at the boundary. Let us impose Dirichlet conditions $\Theta(0)=\theta_{0}$ at $x=0$ and $\Theta(1)=\theta_{1}$ at $x=1$. The condition for stationarity of the energy-density then leads to 
$\operatorname{div}(\rho \Theta \nabla \Theta)=0$; these two remarks can be expressed as

$$
\begin{aligned}
(\Theta \rho)^{\prime}+\rho V^{\prime} & =0 \\
\left(\Theta \Theta^{\prime} \rho\right)^{\prime} & =0 .
\end{aligned}
$$

The second can be solved exactly for $\rho$ in terms of $\Theta$. We assume that $\Theta(x)$ and $\Theta(x)^{\prime}$ have no zeros in $[0,1]$. This gives

$$
\rho=k /\left(\Theta \Theta^{\prime}\right),
$$

where $k$ is the constant of integration, assumed not to be zero. Eliminating $\rho$ from the first equation, we get

$$
\Theta \Theta^{\prime \prime}-\Theta^{\prime} V^{\prime}=0
$$

This can be solved exactly for $\Theta$ if $\mathrm{V}(\mathrm{x})$ is linear, and $\theta_{0}=\theta_{1}$. Thus suppose that

$$
V(x)=g x
$$

as in a constant gravitational field. Then we get the obvious solution $\Theta(x)=$ $\theta_{0}=$ const., and this leads to the solution for the density:

$$
\rho(x)=C \exp \left\{-g x / \Theta_{0}\right\}
$$

This is just that given by the Boltzmann factor, exhibiting the usual exponential fall-off of the density with height. This result shows that the equations are on the right track.

We can find many exact solutions by choosing $\Theta$, satisfying the boundary conditions, instead of choosing $V$. We then just need to solve the firstorder equation (13) for $V(x)$. Not all choices of $\Theta(x)$ lead to nonsingular equations. For example, if we try to satisfy boundary conditions in which $\theta_{0}=\theta_{1}$, then a non-negative differentiable function $\Theta(x)$ must have a zero for $\Theta^{\prime}$ somewhere in the interval $[0,1]$. Then both $\rho$ and the force $-\nabla V$ must be infinite somewhere in the interval. It follows that the only continuous stationary solution with $\theta_{0}=\theta_{1}$, and $\Theta^{\prime}$ finite, is constant in $x$. It is easy find stationary solutions satisfying other boundary conditions; for example choose $\Theta(x)=(x+1)^{2}$; then we have a stationary solution

$$
\begin{aligned}
\rho(x) & =k(x+1)^{-3} \\
\Theta(x) & =(x+1)^{2} \\
V(x) & =x+x^{2} / 2 .
\end{aligned}
$$


This satisfies the equations together with the conditions that $j_{c}(x)=0$ for all $x$, and the boundary conditions $\Theta(0)=1, \Theta(1)=4$. The energy-flow, however is not zero, but is $-4 \lambda$. This flows in at $x=1$ and out at $x=0$, and is constant throughout the region. We have a driven stationary system far from equilibrium. We can follow the production of entropy explicitly; heat enters the system at a high temperature, $\Theta=4$ and leaves at a low temperature, $\Theta=1$. Heat leaves at the same rate as it enters. So the system receives entropy at the rate $Q / \Theta(1)=4 \lambda / 4=\lambda$, and ejects it at the rate $Q / \Theta(0)=4 \lambda$, a net production rate of $3 \lambda$. Where is the location of this entropy production? If we use the Onsager formalism, we can write the entropy production as 15], equation (4.11)

$$
\Theta \dot{S}=\int j_{c} \cdot X^{c} / \Theta+\int j_{e} \cdot X^{e} / \Theta
$$

Here, $X^{c}$ and $X^{e}$ are the thermodynamic forces, given by [15] (4.13) and (4.14). We only need

$$
X^{e} / \Theta=\nabla(1 / \Theta)
$$

since $j_{c}=0$. The entropy produced by the system is therefore

$$
\dot{S}=\int_{0}^{1} j_{e} \cdot X^{e} / \Theta d x=-4 \lambda\left[(x+1)^{-2}\right]_{0}^{1}=3 \lambda .
$$

At a stationary distribution, there is no change in the microscopic probability distribution with time, so it seems that the von Neumann entropy (from which the entropy in [15] is derived) must be independent of time. This is true, and is derived in eq. (4.10) in [15]. In getting the Onsager form eq. (19), we discarded the boundary term, which if kept would appear as the loss of entropy by the system at the rate $3 \lambda$, due to receiving hot heat and emitting cooler heat. The total change in the entropy is therefore zero, as befits a time-independent probability distribution. The environment, however, has an increase in entropy at the rate of $3 \lambda$.

It is easy to construct many more examples.

\section{Analysis of stability in the field-free case}

We limit this section to the study of the case, in one dimension, on the interval $[0,1]$, where $V=0$, and $\Theta(0)=\Theta(1)$. We choose to write the dynamics in terms of the fields $\mathcal{E}:=\rho \Theta$ and $\Theta$; these have simple boundary 
conditions, Neumann and Dirichlet at the points $x=0$ and $x=1$, respectively. Note that at the boundary, $\nabla \mathcal{E}=0$, because the material current, which is $j_{c}=-\lambda \nabla \mathcal{E}$, vanishes on the boundary. The energy current is $2 \Theta j_{c}-2 \lambda \rho \Theta \nabla \Theta=-2 \lambda \nabla(\Theta \mathcal{E})$. We can recover $\rho=\mathcal{E} / \Theta$ from these fields.

The equations of continuity, (2) and (3) then become

$$
\begin{aligned}
\frac{\dot{\mathcal{E}}}{\Theta}-\frac{\mathcal{E} \dot{\Theta}}{\Theta^{2}} & =\lambda \nabla^{2} \mathcal{E} \\
\dot{\mathcal{E}} & =2 \lambda \nabla^{2}(\Theta \mathcal{E}) .
\end{aligned}
$$

These can be written

$$
\begin{aligned}
\dot{\Theta} & =\frac{2 \lambda \Theta}{\mathcal{E}}(\Theta \mathcal{E})^{\prime \prime}-\lambda \frac{\Theta^{2}}{\mathcal{E}} \mathcal{E}^{\prime \prime} \\
\dot{\mathcal{E}} & =2 \lambda(\Theta \mathcal{E})^{\prime \prime} .
\end{aligned}
$$

These clearly have the stationary solution $\Theta(x)=\Theta(0)=\Theta_{0}, \mathcal{E}(x)=\mathcal{E}(0)=$ $\mathcal{E}_{0}$, for all $x \in[0,1]$. To test the stability of this solution, we put

$$
\Theta(x, t)=\Theta_{0}+\theta(x, t) ; \quad \mathcal{E}(x, t)=\mathcal{E}_{0}+e(x, t)
$$

where $\theta(x, t)$ and $e(x, t)$ are small functions, so that we ignore powers higher than the first, of these and their derivatives. Up to the first order, the equations become

$$
\begin{aligned}
\dot{e} & =2 \lambda\left[\left(\Theta_{0}+\theta\right)\left(\mathcal{E}_{0}+e\right)\right]^{\prime \prime} \\
& =2 \lambda\left[\mathcal{E}_{0} \theta^{\prime \prime}+\Theta_{0} e^{\prime \prime}\right]
\end{aligned}
$$

Also

$$
\begin{aligned}
\dot{\theta}= & \frac{2 \lambda\left(\Theta_{0}+\theta\right)}{\mathcal{E}_{0}+e}\left[\left(\Theta_{0}+\theta\right)\left(\mathcal{E}_{0}+e\right)\right]^{\prime \prime} \\
& -\frac{\lambda\left(\Theta_{0}+\theta\right)^{2}}{\mathcal{E}_{0}+e} e^{\prime \prime} \\
= & \frac{2 \lambda \Theta_{0}}{\mathcal{E}_{0}}\left[\mathcal{E}_{0} \theta^{\prime \prime}+\left(\Theta_{0} / 2\right) e^{\prime \prime}\right] .
\end{aligned}
$$

The linearized version of the equations can therefore be summarised as

$$
\left(\begin{array}{c}
\dot{e} \\
\dot{\theta}
\end{array}\right)=2 \lambda \Theta_{0}\left[\begin{array}{cc}
1 & \gamma \\
1 /(2 \gamma) & 1
\end{array}\right]\left(\begin{array}{l}
e^{\prime \prime} \\
\theta^{\prime \prime}
\end{array}\right):=L\left(\begin{array}{c}
e^{\prime \prime} \\
\theta^{\prime \prime}
\end{array}\right)
$$


where $\gamma=\mathcal{E}_{0} / \Theta_{0}$. The boundary conditions are $e^{\prime}(0)=e^{\prime}(1)=0=\theta(0)=$ $\theta(1)$. A necessary condition for the stability of the solution $\left(\mathcal{E}_{0}, \Theta_{0}\right)$ is that the non-zero part of the spectrum of the operator

$$
M \otimes \frac{d^{2}}{d x^{2}}=\left[\begin{array}{cc}
1 & \gamma \\
1 /(2 \gamma) & 1
\end{array}\right] \otimes \frac{d^{2}}{d x^{2}}
$$

entering eq. (27) lies in the open left-half plane. We shall prove this, and show that the spectrum is discrete and negative, of finite multiplicity. Zero is in the spectrum, since $e(x)=$ non-zero constant, $\theta(x)=0$ is the corresponding eigenfunction. This perturbation corresponds to moving from one stationary state to another, with a different total number of particles.

$M$ is not symmetric, but is diagonalisable by a similarity, by Sylvester's criterion. In fact, there is a one-parameter family of diagonalising matrices; for, if $S M S^{-1}=\widehat{M}$ is diagonal, then the one-parameter family of diagonal matrices $B(\beta)=\operatorname{diag}[\beta, 1 / \beta]$ commute with $\widehat{M}$, and so leave it diagonal: $B \widehat{M} B^{-1}=\widehat{M}$. In general, such a transformation leaves us with non-self-adjoint boundary conditions. These can be expressed as a singular perturbation of the Laplacian, in the manner of [1]. This monograph, however, only deals with the self-adjoint case. This extra one-parameter family of diagonalising similarities can be used to ensure that the boundary conditions define a symmetric Dirichlet form, by a making judicious choice of $\beta$. Note that it is enough to diagonalise $(M-I)$; this leads to the special case, $\alpha=1 / 2$, of the problem of finding all matrices $S$ such that

$$
S=\left[\begin{array}{ll}
a & b \\
c & d
\end{array}\right]
$$

and

$$
S\left[\begin{array}{cc}
0 & \gamma \\
\alpha / \gamma & 0
\end{array}\right] S^{-1}=\operatorname{diag}\left[\lambda_{1}, \lambda_{2}\right]
$$

Direct calculation immediately gives the conditions for the off-diagonal terms in $\widehat{M}$ to vanish:

$$
\begin{aligned}
b^{2} \alpha & =a^{2} \gamma^{2} \\
\alpha d^{2} & =c^{2} \gamma^{2} .
\end{aligned}
$$

To avoid the vanishing of $\operatorname{det} S$ we have to choose opposite signs in the square-roots of these equations, for example

$$
\begin{aligned}
b & =a \gamma \alpha^{-1 / 2} \\
d & =-c \gamma \alpha^{-1 / 2} .
\end{aligned}
$$


Then $\widehat{M}=\operatorname{diag}\left[1+\alpha^{1 / 2}, 1-\alpha^{1 / 2}\right]$. We notice that this is independent of the value of $\gamma$. If we can show that the operator $\widehat{M} \otimes d^{2} / d x^{2}$ is a Dirichlet form, it is negative since $\alpha=1 / 2<1$. For this to be the case, the boundary conditions must be self-adjoint, so that the boundary terms that arise in the integration by parts vanish for functions in the domain of the operator. Let

$$
\left(\begin{array}{c}
\hat{e} \\
\hat{\theta}
\end{array}\right)=S\left(\begin{array}{l}
e \\
\theta
\end{array}\right),
$$

where

$$
S=\left(\begin{array}{cc}
a & a \gamma \alpha^{-1 / 2} \\
c & -c \gamma \alpha^{-1 / 2}
\end{array}\right)
$$

Thus,

$$
\begin{aligned}
& \hat{e}=a e+a \gamma \alpha^{-1 / 2} \theta \\
& \hat{\theta}=c e-c \gamma \alpha^{-1 / 2} \theta .
\end{aligned}
$$

Then

$$
\begin{aligned}
(\hat{e}, \hat{\theta}) \hat{M}\left(\begin{array}{c}
\hat{e}^{\prime \prime} \\
\hat{\theta}^{\prime \prime}
\end{array}\right)= & \left(1+\alpha^{1 / 2}\right)\left\langle\hat{e}, \hat{e}^{\prime \prime}\right\rangle+\left(1-\alpha^{1 / 2}\right)\left\langle\hat{\theta}, \hat{\theta}^{\prime \prime}\right\rangle \\
= & -\left(1+\alpha^{1 / 2}\right)\left\langle\hat{e}^{\prime}, \hat{e}^{\prime}\right\rangle-\left(1-\alpha^{1 / 2}\right)\left\langle\hat{\theta}^{\prime}, \hat{\theta}^{\prime}\right\rangle \\
& +\left(1+\alpha^{1 / 2}\right)\left[\hat{e} \hat{e}^{\prime}\right]_{0}^{1}+\left(1-\alpha^{1 / 2}\right)\left[\hat{\theta} \hat{\theta}^{\prime}\right]_{0}^{1}
\end{aligned}
$$

The boundary terms which must vanish are thus

$$
\begin{aligned}
&\left(1+\alpha^{1 / 2}\right)\left[\left(a e+a \gamma \alpha^{-1 / 2} \theta\right)\left(a e^{\prime}+a \gamma \alpha^{-1 / 2} \theta^{\prime}\right)\right]_{0}^{1}+ \\
&+\left(1-\alpha^{1 / 2}\right)\left[\left(c e-c \gamma \alpha^{-1 / 2}\right)\left(c e^{\prime}-c \gamma \alpha^{-1 / 2} \theta^{\prime}\right)\right]_{0}^{1} \\
&=\left\{\left(1+\alpha^{1 / 2}\right) \gamma \alpha^{-1 / 2} a^{2}-\left(1-\alpha^{1 / 2}\right) \gamma \alpha^{-1 / 2} c^{2}\right\}\left[e \theta^{\prime}\right]_{0}^{1} .
\end{aligned}
$$

For this to vanish for all $e, \theta$ in the domain, we need to be able to choose $\left(1+\alpha^{1 / 2}\right) a^{2}=\left(1-\alpha^{1 / 2}\right) c^{2}$ without making $S$ singular; this is possible. We conclude that our operator $L$ is similar to a negative self-adjoint operator with compact resolvent, and so has non-positive discrete spectrum. The domain of the operator is

$$
\left\{(e, \theta) \in W^{2,2}: e^{\prime}(0)=e^{\prime}(1)=0 \text { and } \theta(0)=\theta(1)=0\right\} .
$$


In order to solve the eigenvalue problem, we try

$$
\begin{aligned}
& \hat{e}(x, t)=A \exp \{-\omega t\} \cos \left(k_{1} x\right)+B \exp \{-\omega t\} \sin \left(k_{1} x\right) \\
& \hat{\theta}(x, t)=C \exp \{-\omega t\} \cos \left(k_{2} x\right)+D \exp \{-\omega t\} \sin \left(k_{2} x\right) .
\end{aligned}
$$

We have proved above that $\omega$, an eigenvalue of $-L$, is nonnegative; $k_{1}$ and $k_{2}$ must be chosen to satisfy

$$
\frac{\omega}{2 \lambda \Theta(0)}=\left(1+\alpha^{1 / 2}\right) k_{1}^{2}=\left(1-\alpha^{1 / 2}\right) k_{2}^{2} .
$$

To fit the boundary conditions, we can write $(\hat{e}, \hat{\theta})$ in terms of $(e, \theta)$ and then use the given conditions. However, since a similarity does not alter the eigenvalue (only the eigenfunctions) we can avoid the complication of choosing $S$ so that the boundary terms vanish, and instead use any convenient matrix that diagonalises $M$. We choose

$$
S=\left(\begin{array}{cc}
1 & \alpha^{-1 / 2} \\
-1 & \alpha^{-1 / 2}
\end{array}\right)
$$

This gives us the boundary conditions

$$
\begin{aligned}
\hat{e}^{\prime}-\hat{\theta}^{\prime} & =0, & & \text { at } x=0, x=1 \\
\hat{e}+\hat{\theta} & =0, & & \text { at } x=0, x=1 .
\end{aligned}
$$

By imposing this condition, we get a linear homogeneous system of equations for $A, B, C, D$, and the vanishing of the determinant gives us a holomorphic trancendental equation for $\omega$. Note that the eigenvalues do not depend on $\gamma$, the ratio of $\mathcal{E}_{0}$ to $\Theta_{0}$. We have computed the eigenvalues $\omega$ of $-L$, and indeed, they are all non-negative.

In 12] it is proved that the semigroup $U(t)=\exp \{L t\}$ defined by a sectorial operator $L$ with range in the left half-plane is a contraction semigroup in $L^{2}$. Here, however, the conditions for the theorem fail, and the best we can do in $L^{2}$ is to get the inequality for $u$ orthogonal to the one-dimensional subspace of stationary solutions:

$$
\|\exp \{L t\} u\|_{2} \leq \exp \left\{-\lambda_{1} t\right\}\|S\|\left\|S^{-1}\right\|\|u\|_{2}
$$

where $\lambda_{1}$ is the smallest non-zero eigenvalue of $-L$. Since $\|S\|\left\|S^{-1}\right\|>1$ in our model, the system is not obviously stable for small $t$. This problem does not arise for an isolated system, in which there is no flow of heat or material 
at the boundary; this gives us Neumann conditions on the boundary for both $\nabla \mathcal{E}$ and $\nabla \Theta$, which is a self-adjoint condition. Then the numerical range of $M \otimes d^{2} / d x^{2}$ is determined by its symmetric part, which is non-positive.

\section{Acknowledgements}

The author would like to thank E. B. Davies and L. Boulton for very useful discussions, and T. Nadzieja for reading the manuscript.

\section{References}

[1] S. Albeverio and P. Kurosov, "Singular Perturbations of Differential Operators", Cambridge University Press, 2000.

[2] P. Biler and J. Dolbeault, Long time behaviour of solutions to Nernst-Planck and Debye-Hückel drift-diffusion systems, Annales Henri Poincaré, 1, 461-472, 2000.

[3] P. Biler, J. Dolbeault, M. J. Esteban and G. Karch, Stationary solutions, intermediate asymptotics and large-time behaviour of type II Streater's models, Advances in Differential Equations, 6, 461-480, 2001.

[4] P. Biler, J. Dolbeault and P. Markowich, Large time asymptotics of nonlinear drift-diffusion systems with Poisson coupling, Ceremade \# 9932 (1999); to appear in Transport Theory and Statistical Physics.

[5] P. Biler, W. Hebisch and T. Nadzieja, The Debye system: existence and long time behavior of solutions, Nonlinear Analysis, TMA, 23, 1189-1209, 1994.

[6] P. Biler, A. Krzywicki and T. Nadzieja, Self-interaction of Brownian particles coupled with thermodynamic processes, Reports on Mathematical Physics, 42, 359-372, 1998.

[7] P. Biler and T. Nadzieja, A class of nonlocal parabolic problems occurring in statistical mechanics, Colloq. Math., 66, 131-145, 1993.

[8] P. Biler and T. Nadzieja, Existence and nonexistence of solutions for a model of gravitational interaction of particles I, Colloq. Math., 66, 319-334, 1994. 
[9] P. Biler and T. Nadzieja, A nonlocal singular parabolic problem modelling gravitational interaction of particles, Advances in Differential Equations, 3, 177-199, 1998.

[10] S. Chapman, Philosophical Transactions of the Royal Society, A213, 433-, 1912.

[11] D. Enskog, Physikalischer Zeitsschrift, 12 56-,533-, 1911.

[12] T. Kato, "Perturbation Theory of Linear Operators", Springer-Verlag, 1966.

[13] A. Krzywicki and T. Nadzieja, Steady states for a model of interacting particles, Applied Math. Letters, 13, 113-117, 2000.

[14] T. Nadzieja and A. Raczynski, Radially symmetric solutions of the Poisson-Boltzmann equation with a given energy, to appear in Appl. Math. (Warsaw).

[15] R. F. Streater, The Soret and Dufour effects in statistical dynamics, Proc. Roy. Soc., 456, 205-221, 2000. Lanl archive: math-ph/9910043

[16] R. F. Streater, A gas of Brownian particles in statistical dynamics, Journal of Statistical Physics, 88, 447-469, 1997.

[17] R. F. Streater, Dynamics of Brownian particles in a potential, Journal of Mathematical Physics, 38, 4570-4575, 1997.

[18] R. F. Streater, Nonlinear heat equations, Reports on Mathematical Physics, 40, 557-564, 1997.

[19] R. F. Streater, A model of dense fluids, Banach Center Publications, 43, 381-393, 1998.

[20] R. F. Streater, Onsager relations in statistical dynamics, Open Systems and Information Dynamics, 6, 87-100, 1999. Kluwer.

[21] R. Wojnar, On nonlinear heat equations and diffusion in porous media, Reports on Mathematical Physics, 44, 291-300, 1999. 